\title{
Expanding the horizon of research into the pathogenesis of the white matter diseases: Proceedings of the 2021 Annual Workshop of the Albert Research Institute for White Matter and Cognition
}

\author{
Shawn N. Whitehead · Askiel Bruno · Jeffrey M. Burns · S. Thomas Carmichael • Anna Csiszar • \\ Jodi D. Edwards • Fanny M. Elahi • Giuseppe Faraco • Douglas B. Gould • Deborah R. Gustafson • \\ Vladimir Hachinski • Gary Rosenberg • Farzaneh A. Sorond • Andy Y. Shih • Kai Hei Tse • \\ Zoltan Ungvari · Donna M. Wilcock · Kristen L. Zuloaga $\cdot$ Frank C. Barone
}

Received: 13 September 2021 / Accepted: 14 September 2021 / Published online: 4 October 2021

(C) The Author(s), under exclusive licence to American Aging Association 2021

\begin{abstract}
White matter pathologies are critically involved in the etiology of vascular cognitive impairment-dementia (VCID), Alzheimer's disease (AD), and Alzheimer's disease and related diseases (ADRD), and therefore need to be considered a treatable target ( Roseborough A, Hachinski V, Whitehead S. White matter degeneration - a treatable target? Roseborough et al. JAMA Neurol [Internet]. 2020 Apr 27;77(7):793-4, [1] . To help address this often-
\end{abstract}

S. N. Whitehead $(\bowtie)$

Department of Anatomy and Cell Biology, Western

University, London, ON N6A 3K7, Canada

e-mail: shawn.whitehead@schulich.uwo.ca

\section{A. Bruno}

Department of Neurology, Medical College of Georgia

at Augusta University, Augusta, GA 30912, USA

J. M. Burns

Department of Neurology, University of Kansas Medical Center, Kansas City, KS, USA

\section{S. T. Carmichael}

Department of Neurology, David Geffen School

of Medicine at UCLA, Los Angeles, CA 90095, USA

\section{A. Csiszar $\cdot$ Z. Ungvari}

Vascular Cognitive Impairment and Neurodegeneration Program, Oklahoma Center for Geroscience and Healthy Brain Aging, Department of Biochemistry and Molecular Biology, University of Oklahoma Health Sciences Center, Oklahoma City, OK 73104, USA missed area of research, several workshops have been sponsored by the Leo and Anne Albert Charitable Trust since 2015, resulting in the incorporation of "The Albert Research Institute for White Matter and Cognition" in 2020. The first annual "Institute" meeting was held virtually on March 3-4, 2021. The Institute provides a forum and workspace for communication and support of the advancement of white matter science and research to better understand the

\author{
A. Csiszar · Z. Ungvari \\ International Training Program in Geroscience, Doctoral \\ School of Basic and Translational Medicine/Department \\ of Translational Medicine, Semmelweis University, \\ Budapest, Hungary \\ J. D. Edwards \\ University of Ottawa Heart Institute, Ottawa, Canada \\ J. D. Edwards \\ School of Epidemiology and Public Health, University \\ of Ottawa, Ottawa K1G 5Z3, Canada \\ F. Elahi \\ Memory and Aging Center, UCSF Weill Institute \\ for Neurosciences, 675 Nelson Rising Lane, Suite 190, \\ San Francisco, CA 94158, USA \\ G. Faraco \\ Feil Family Brain and Mind Research Institute, Weill \\ Cornell Medicine, New York, NY 10065, USA
}


evolution and prevention of dementia. It serves as a platform for young investigator development, to introduce new data and debate biology mechanisms and new ideas, and to encourage and support new research collaborations and directions to clarify how white matter changes, with other genetic and health risk factors, contribute to cognitive impairment. Similar to previous Albert Trust-sponsored workshops (Barone et al. in J Transl Med 14:1-14, [2]; Sorond et al. in GeroScience 42:81-96, [3]), established expert investigators were identified and invited to present. Opportunities to attend and present were also extended by invitation to talented research fellows and younger scientists. Also, updates on institute-funded research collaborations were provided and discussed. The summary that follows is a synopsis of topics and discussion covered in the workshop.

Keywords White matter - Cognition .

Neurodegenerative disease/injury $\cdot$ AD $\cdot$ VCID

\section{Introduction}

According to the World Health Organization, in the year 2050 one in five of the world's population will be over the age of 60, with approximately 115 million individuals affected by dementia [4]. In addition

\section{B. Gould}

Departments of Ophthalmology and Anatomy, and Institute for Human Genetics, School of Medicine, University of California, San Francisco 94143, USA

\section{R. Gustafson}

Department of Neurology, Section for NeuroEpidemiology, State University of New York Downstate Health Sciences University, New York, Brooklyn 11203, USA

\section{Hachinski}

Department of Clinical Neurological Sciences, Western University, London, ON N6A 5C1, Canada

\section{G. Rosenberg}

UNM Health Sciences Center, University of New Mexico, Albuquerque, NM 87106, USA

\section{F. A. Sorond}

Department of Neurology, Northwestern University, Chicago, IL, USA

A. Y. Shih

Center for Developmental Biology and Regenerative

Medicine, Seattle Children's Research Institute; to this number, nearly $20 \%$ of elderly individuals will be predicted to suffer from mild cognitive impairment, which occurs years prior to dementia [5]. To date, most therapies aim to protect/preserve at-risk neurons, but very few target the brain white matter, which makes up half of the brain and can be a critical site of prodromal disease. While neuronal loss within the gray matter is a widely studied feature of aging, the parallel (and potentially preceding) loss of white matter has garnered less attention. White matter is well recognized to be particularly vulnerable to cerebrovascular injury $[6,7]$. Accordingly, white matter pathology is commonly seen in VCID [8], most often presenting with cognitive slowing and executive dysfunction $[9,10]$. This vulnerability has been attributed, in part, to the nature of cerebral vascular anatomy [11-15]. However, the active and contributing role of glial cells in white matter vascular injury has been demonstrated by the inhibition and modulation of these cells in recent experimental models [16-22]. Additionally, impairments in white matter structure and function are a core feature of aging, are one of the earliest pathological features of Alzheimer disease (AD), and are a significant risk factor for cerebrovascular disease [1,23].

White matter changes are detectable on neuroimaging, beginning in midlife with a prevalence as high as $50 \%$ by the individuals in their $40 \mathrm{~s}$ [24].

Department of Pediatrics; Department of Bioengineering, University of Washington, Seattle, WA, USA

K. H. Tse

Department of Health Technology and Informatics, The Hong Kong Polytechnic University, Kowloon, Hong Kong

D. M. Wilcock

Sanders-Brown Center on Aging; Department of Neurology, Department of Behavioral Science, University of Kentucky, Lexington, KY 40536, USA

\section{K. L. Zuloaga}

Department of Neuroscience and Experimental Therapeutics, Albany Medical College, Albany, NY 12208, USA

F. C. Barone

Department of Neurology, SUNY Downstate Health Sciences University, Brooklyn, NY 11203, USA 
Traditionally, white matter changes are detected using magnetic resonance imaging (MRI) using T2 weighted fluid attenuated inversion recovery (FLAIR), appearing as white matter hyperintensities (WMH). The etiology and development of WMH are heterogeneous, but usually involve a loss of myelin and axon density, the primary end-stage pathological correlates of WMH [25]. Within both gray and white matter, glial cells present critical targets for therapeutic intervention and neuroprotection [26-28]. This pertains to VCID as well as Alzheimer disease (AD), as the roles of both cerebrovascular and executive dysfunction are now increasingly appreciated as common factors of $\mathrm{AD}$ [8, 29-33]. Additionally, investigation of the neurovascular unit (NVU) in neurodegenerative disease has identified an important intersection between cerebrovascular and glial pathology [34].

\section{Synopsis of 3-4 March 2021 Albert Research Institute for White Matter and Cognition workshop}

The recent workshop scientific program was organized into four topics: (i) cerebrovascular disease: models, markers, and mediator mechanisms (Faraco, Zuloaga, Carmichael, Tse, Shih); (ii) epidemiology, aging, and neurovascular dysregulation (Gufstafson, Ungvari, Elahi, Csiszar); (iii) white matter, brain imaging, and biomarkers (Hachinski, Wilcock, Gould, Edwards); and (iv) white matter, brain protection, and mechanisms (Burns, Bruno, Seshadri). Due to the ongoing COVID-19 pandemic at this time, the meeting was held virtually over 2 days via Zoom, and individual synopses of the talks are provided below.

\section{Gut flora, immune-mediated neurovascular dysfunction and tau accumulation}

\section{Guiseppe Faraco}

The intestinal flora is essential for the development of gut-associated lymphoid tissue and for maintaining the homeostasis of the immune system. IL-17-producing T-helper lymphocytes (Th17 cells) are particularly abundant in GALT and play a major role in the cognitive dysfunction associated with high-salt diet in mice [35, 36]. This harmful effect depends on circulating IL17 acting on cerebral endothelial cells to induce a deficit in endothelial nitric oxide, leading to tau phosphorylation and aggregation. These observations raise the possibility that gut dysbiosis, resulting in increased Th17 differentiation, may lead to cognitive impairment through tau. To test this hypothesis, we co-housed C57BL6 mice from Jackson Laboratory (Jax) with C57BL6 mice from Taconic Biosciences, the microbiota of which is notable for the presence of segmented filamentous bacteria, potent inducers of Th17 cells. Owing to their coprophagic behavior, sharing the same cage resulted in colonization of Jax mice by SFB and, in turn, increased IL17 mRNA levels in the distal small intestine. Circulating levels of IL-17 were also increased in Jax mice after cohousing. Neurovascular coupling and endotheliumdependent vasodilation were impaired, and brain tau phosphorylation was increased in Jax mice. Furthermore, Jax mice showed deficits in spatial memory assessed by the Barnes maze. Although additional studies are needed to further elucidate the underlying mechanisms, these studies unveil a previously unrecognized link between changes in gut microbiota and tau phosphorylation and may provide new insights in the pathobiology of $\mathrm{AD}$ and other tauopathies with potential therapeutic relevance.

\section{Sex differences in metabolic and hormonal contributions to VCID}

\section{Kristen L. Zuloaga}

Mid-life metabolic disease causes cerebrovascular dysfunction and is a risk factor for VCID. The effect of metabolic disease on VCID risk is even greater for women compared to men. In line with this clinical data, using mouse models of metabolic disease (chronic high fat diet) and VCID (chronic cerebral hypoperfusion), we have found that high-fat diet causes greater metabolic impairment and a wider array of cognitive deficits in middle-aged females compared to males [37]. We have found that metabolic disease severity is more strongly associated with white matter damage, astrogliosis [38], and reduced neurogenesis [39] in females compared to 
males. The increased susceptibility in females may be related to endocrine aging. Women are unique in that they go through menopause, with loss of estradiol. Estradiol has protective effects on white matter, gray matter, the vasculature, and metabolism. Menopause accelerates mid-life risk factors for dementia, by increasing risk for cardiovascular and metabolic diseases. Despite the fact that nearly all women with VCID are post-menopausal, the influence of menopause on VCID and underlying pathology is a critical gap in knowledge. Using an accelerated ovarian failure model of menopause in mice, we have recently found that longer time in menopause is associated with increased memory impairment in a mouse model of VCID. Our next steps will be to determine if the underlying pathologies are affected. We anticipate these studies will provide mechanistic insight that will facilitate future interventions to decrease the burden of VCID in the high-risk population of post-menopausal women with metabolic disease.

\section{Neural repair after white matter stroke}

\section{S. Thomas Carmichael}

White matter stroke progresses into adjacent white matter over time, and white matter lesions accumulate to produce vascular dementia [40, 41]. In inflammatory diseases of white matter, such as multiple sclerosis, there is a process of white matter repair early in the course of disease, produced through oligodendrocyte progenitor cell (OPC) responses [42]. OPCs sense the lesion, and proliferate and differentiate into mature oligodendrocytes. Such a process does not happen in white matter stroke, despite an initial similar size and location of ischemic white matter lesions as compared to multiple sclerosis plaques [43, 44]. In a mouse model of white matter stroke/vascular dementia, we have identified the molecular responses of OPCs as a function of age (young vs aged adult), time after stroke and with stroke in comparison to other types of white matter lesions. These data indicate a specific deficit in subtypes of OPCs with normal aging, impairment in OPC differentiation with age and an inflammatory OPC that occurs in stroke. In a separate series of questions, behavioral action and neural activity promote white matter structure and function in a variety of contexts. Can specific types of neurorehabilitation promote white matter repair in white matter stroke/vascular dementia? If so, is there a specific molecular system that is induced by neurorehabilitation that might serve as a therapeutic target? We will show evidence for the effect of neuro-rehabilitative therapies on white matter repair in mouse white matter stroke.

\section{TRIM47 in white matter hyperintensity: from the population to the cell}

\section{K.H. Tse}

WHM represent a radiopathology highly associated with cognitive declines in aging, vascular dementia, and Alzheimer's disease. Different genome-wide association studies (GWAS) commonly identified single nucleotide polymorphisms (SNPs) at the 17q25 locus spanning the genes of Tripartite motif (TRIM) family, a group of E3 ubiquitin ligase proteins, associated with WMH. In particular, multiple SNPs were detected in TRIM47 [45, 46]. We wished to test the hypothesis that TRIM47 is a critical protein that contributes to the pathogenesis of WMH. However, as the expression and function of TRIM47 in the brain remain obscure, we undertook to establish the basic characteristics and roles of TRIM47 in the pathogenesis of WMH. In the exploration of RNA-seq datasets of human tissues and mouse brain, we first confirmed that TRIM47 is enriched in the cerebral cortex, where it is highly expressed by brain endothelial cells. Our immunohistochemistry experiments validated that TRIM47 proteins are specifically localized in endothelial cells of postmortem human brain tissues. In an acute mouse model of WMH (BCAS; bilateral common carotid arteries stenosis), minimal changes in BCAS were found, but a significant upregulation of Trim 47 was detected in hAPOE4 mice (humanized APOE4) correlated with a heretofore unreported propensity toward periventricular WMH. We have also investigated the change of TRIM47 on a murine brain-derived endothelial cell model under an ischemic-like conditions. While TRIM47 was unaffected by oxidative stress, it was upregulated by hypoglycemic conditions at the mRNA level. Intriguingly, genetic silencing of Trim47 significantly increased the expression of LC3 punta and the formation of vacuoles in endothelial cells and exaggerated 
their responses to hypoglycemia, where confocal microscopy revealed a proximity between LC3 and TRIM47. Structural analysis revealed the existence of a LC3-interacting motif (LIR) in TRIM47, where their putative interaction with LC3 was found in a protein-protein interaction model in silico and observed by super-resolution microscopy. Together, the present study characterized the expression pattern and the potential function of TRIM47 in mouse and human brain. Importantly, our preliminary data connects TRIM47 to autophagy, a key cellular mechanism underlying cerebrovascular remodeling in the aging brain. These findings will help us to understand the cell biology underlying WMH pathogenesis and warrant further investigation.

\section{Deep multi-photon imaging of capillary drainage at gray-white matter interface in mice}

\section{Andy Y. Shih}

Our understanding of brain microcirculation mostly comes from studying arteriolar perfusion. However, blood drainage through venules is equally important in regulating cerebral blood flow. Properties of cerebral venules can change dramatically during cerebrovascular disease and aging. Understanding the regulation of blood drainage in and near white matter tracts is critical in elucidating the basis of white matter degeneration in early stages of ADRD. We studied principal cortical venules (PCVs), massive ascending venules extending from the brain surface to the deepest layers of cortex and underlying white matter. Deep in vivo two-photon imaging was used to perform structural and functional characterization of PCV vascular networks in adult (5-7 months) and aged mice (22-24 months). The study reveals cortical layer specific changes in the structural and hemodynamic properties of PCV capillary networks. Namely, capillaries draining cortical layer 6 and the superficial white matter exhibit reduced diameter, blood cell flux, and velocity, as well as increased lengths and tortuosity with advanced age. We also show that in vivo three-photon imaging is applicable for interrogation of microvascular networks in deeper aspects of the white matter. This novel imaging study of healthy adult and aged mice provides an experimental foundation for studies of venular dysfunction as a mechanism of impaired cerebral blood flow and white matter degeneration in ADRD.

\section{Epidemiology informs interventions for VCID}

\section{Deborah R. Gustafson}

Observational epidemiology studies show differential associations between vascular risk factors and both clinical and neuropathological brain aging outcomes. Associations between individual vascular risk factors and individual symptoms-neuropathology of VCID, ADRD, and cerebrovascular small vessel disease (CSVD) across diverse, global populations facilitate identification of disease mechanisms, susceptible populations, and intervention targets. Greater attention to "fit for purpose and fit for community" results in better planning of interventions for VCID, ADRD, and CSVD [47]. Peripheral measures of adiposity as the vascular risk factor and exposure in relation to brain health are provided using two examples. The first example involves CSVD brain imaging outcomes in the RUN DMC study of 503 adults with CSVD, age $\geq 50$ years. RUN DMC reported differences in cross-sectional versus longitudinal adiposity associations, in association with volumetric and vascular brain hallmarks of VCID and ADRD. Differences by anthropometric versus metabolic adiposity measures, sex, and survivorship were observed [48]. The second example is unpublished data from the Women's Interagency HIV Study. During middle age, from average age 38-48 years, a single timepoint versus 10-year change in adiposity measures differed in association with neuropsychological performance at 10 years, particularly in HIV-infected women. Translating the observational epidemiology to interventions must consider the following: (1) changing vascular phenotypes over the life course; (2) characteristics of populations at risk; (3) multi-morbidities; (4) duration of exposure(s), such as medications; (5) birth cohort; and (6) genetic susceptibility. Embracing the details will better identify optimal VCID and CSVD intervention strategies $[47,49]$. 


\section{Role of NAD + deficiency in neurovascular and cognitive aging}

\section{Zoltan Ungvari}

Adjustment of cerebral blood flow (CBF) to neuronal activity via neurovascular coupling (NVC) has an essential role in maintenance of healthy cognitive function [50]. Aging increased oxidative stress and cerebral microvascular endothelial dysfunction impairs NVC, contributing to cognitive decline [51]. There is increasing evidence showing that a decrease in $\mathrm{NAD}^{+}$availability with age plays a critical role in age-related cerebral microvascular impairment and that restoring $\mathrm{NAD}^{+}$concentration exerts beneficial effects on the neurovascular unit [52]. When 24-month-old mice were treated with nicotinamide mononucleotide $(\mathrm{NMN})$, a key $\mathrm{NAD}^{+}$intermediate, for 2 weeks, endothelial NO-mediated vasodilation and NVC responses (assessed by measuring CBF responses evoked by contralateral whisker stimulation) were rescued, which was associated with significantly improved cognitive performance. Transcriptome analysis of preparations enriched for cells of the neurovascular unit was performed by RNA-seq. We identified 590 genes differentially expressed in the aged neurovascular unit, 204 of which are restored toward youthful expression levels by NMN treatment. The transcriptional footprint of NMN treatment indicates that increased NAD+levels promote SIRT1 activation in the neurovascular unit, as demonstrated by analysis of upstream regulators of differentially expressed genes as well as analysis of the expression of known SIRT1-dependent genes. Pathway analysis predicts that neurovascular protective effects of NMN are mediated by the induction of genes involved in mitochondrial rejuvenation, anti-inflammatory and anti-apoptotic pathways. These findings are paralleled by the sirtuin-dependent protective effects of NMN on mitochondrial production of reactive oxygen species and mitochondrial bioenergetics in cultured cerebral microvascular endothelial cells derived from aged animals. Thus, a decrease in $\mathrm{NAD}^{+}$availability contributes to age-related microvascular dysfunction, exacerbating cognitive decline. The cerebral microvascular protective effects of NMN highlight the preventive and therapeutic potential of $\mathrm{NAD}^{+}$intermediates as effective interventions in patients at risk for VCID.

\section{New tools for studies of neurovascular aging and dysfunction in humans}

\author{
Fanny Elahi
}

Although vascular risk and disease are known clinical contributors to cognitive impairment and dementia, the cellular and molecular underpinnings of VCID remain more obscure. In our model of white matter disease and VCID pathogenesis, we posit that vascular risk factors and systemic innate immune activation are associated with endothelial injury and inflammation, with phenotypic change in endothelial cells contributing to impairment of the blood-brain barrier (BBB), followed by CNS immune activation and neurodegeneration. To begin testing this multi-component disease model, we have worked on developing an approach that combines specificity for endothelial cells with sensitivity for molecules within pathways of interest. We use sensitive quantitative methods to analyze molecular cargo from plasma endothelial-derived extracellular vesicles (EDE) as a "liquid biopsy" approach of endothelial cells. Using this approach, we have thus far shown (1) that plasma EDE capture molecular cargo that are relevant to brain endothelial cells, PMID: 30483114 and PMID: 32157747 (2) that EDE molecular cargo in aging adults with vascular risk or white matter disease have higher levels of complement factors, PMID: 34376699 and (3) that EDE-complement levels are associated with clinically meaningful outcomes in VCID, such as cognitive processing speed and executive function PMID: 34376699. This approach could provide the much needed cellular specificity in molecular measurements from human biospecimen to allow better translation between mechanistic studies in reductionist systems and molecular dysregulations in complex systems such as human aging and disease.

\section{Potential role of neurovascular senescence in cognitive decline}

\section{Anna Csiszar}

Age-related phenotypic changes of cerebral microvascular endothelial cells lead to dysregulation of 
cerebral blood flow and blood-brain barrier disruption, promoting the pathogenesis of VCID [51, 53]. In recent years, endothelial cell senescence has emerged as a potential mechanism contributing to microvascular pathologies $[54,55]$, opening the avenue to the therapeutic exploitation of senolytic drugs in preclinical studies [56]. To detect senescent endothelial cells in the aging mouse brain, we analyzed 4233 cells in fractions enriched for microvascular endothelial cells and other cells associated with the neurovascular unit obtained from young (3-month-old) and aged (28-month-old) C57BL/6 mice. We defined 13 transcriptomic cell types by deep, single-cell RNA sequencing. We matched transcriptomic signatures of cellular senescence to endothelial cells identified on the basis of their gene expression profile. Our study demonstrates that with advanced aging there is an increased ratio of senescent endothelial cells $(\sim 10 \%)$ in the mouse cerebral microcirculation. Spatial transcriptomics confirmed the increased presence of senescent cells in the white matter as well as in the hippocampus and cortex of the aged mouse brain. To evaluate the efficiency of a translationally relevant senolytic regimen, we treated aged mice with the BCL-2/BCL-xL inhibitor senolytic drug ABT263/ Navitoclax. We found that Navitoclax improves functional hyperemia in aged mice that associates with improved cognitive performance. Our results open the avenue to the therapeutic exploitation of senolytic drugs in multiple age-related cerebrovascular pathologies in preclinical studies.

\section{Leukoaraiosis: 3 decades later}

Vladimir Hachinski

The advent of brain imaging in the 1980s began revealing white matter changes that were promptly equated with "Binswanger disease." However, Binswanger disease is neither Binswanger's nor a disease. We suggested that the white matter changes had diverse causes but a common denominator: decreased density. Thus, we coined the term "leukoaraiosis" (LA) based on ancient Greek: Leuko meaning white and araiosis, less dense. Although it remains true that LA has many causes, the concept of the ambibaric brain helps explain the pathogenesis and symptomology of LA associated with vascular disease. The brainstem, basal ganglia, and thalamus are supplied by short end arteries (primitive brain). Having to step down arterial to capillary pressure makes these arteries a high-pressure system vulnerable to hypertension. By contrast, the arteries that supply the hemispheres (Homo sapiens brain) have long distances to step down the pressure before they supply the brain, making them a low-pressure system. Thus, the brain circulation contains two (ambi) complementary pressure (baric) systems. Damage to the blood vessels of the primitive brain can result in damage to the frontal systems resulting in executive dysfunction, gait slowing, and apathy. Hypertension is a highly prevalent and treatable cause of small vessel disease and the resulting symptomology. LA can be an early warning system for cognitive impairment and stroke and a growingly attractive therapeutic and prevention target.

\section{Placental growth factor as a mediator of small vessel disease}

\section{Donna M. Wilcock}

VCID is an important and understudied cause of cognitive impairment, often co-morbid with Alzheimer's disease and other neurodegenerative conditions. Diffuse white matter disease (DWD) is apparent on MRI, appearing as WMH. It has long been considered to be a consequence of cerebrovascular pathology; more specifically small vessel disease resulting from hypertension and microinfarcts. Much like the pathologic angiogenesis that occurs in retinopathies, pathologic angiogenesis in the brain results in blood-brain barrier leakage and, counterintuitively, tissue ischemia. We have examined plasma and CSF angiogenic mediators in a cohort of 120 research participants who also have a range of cognitive impairments and severity of WMH changes on MRI. We found several members of the vascular endothelial growth factor (VEGF) family, in particular placental growth factor (PIGF), which is significantly associated with WMH growth and cognitive measures, especially executive function measures. We have examined post-mortem autopsy tissue to determine PlGF expression in the human brain, and we found strong immunostaining in the white matter of individuals with small vessel disease, primarily perivascular labeling. Such staining 
was significantly less in the brains of age-matched, pathology-free individuals. We also performed exosome studies in plasma and found that the primary source of PlGF in the plasma is astrocytic, and also there is some PlGF in endothelial exosomes.

\section{Roles for Col4a1 and Col4a2 in cerebral small vessel disease}

\section{Douglas B. Gould}

VCID describes a spectrum of cognitive changes with primary vascular origins and is a significant global health problem. However, a lack of understanding of the pathogenic mechanisms is a barrier to developing specific interventional strategies. CSVD is a leading cause of stroke and VCID and includes WMH, enlarged perivascular spaces (PVS), lacunar infarcts, cerebral microbleeds, and intracerebral hemorrhages. Familial and sporadic forms of CSVD share clinical and radiological features suggesting that monogenic forms of CSVD may represent experimentally tractable models with which to identify pathogenic processes underlying common and chronic disease subtypes. Type IV collagen alpha 1 (COL4A1) and alpha 2 (COL4A2) are extracellular matrix proteins that form heterotrimers deposited into basement membranes of nearly all tissues. Semi-dominant mutations of COLAAI and COLAA2 cause a congenital multisystem disorder that includes highly penetrant cerebrovascular disease and commonly manifests as porencephaly, stroke, WMH, subcortical MB, enlarged PVS, and lacunar infarctions REFS - PMID: 26610912 and 27794444. Importantly, COLAA2 is the most consistently associated with manifestations of CSVD and VCID in genome-wide association studies. Using Col4al mutant mouse models, we observe both mural cell defects during developmental angiogenesis and arterial smooth muscle cell degeneration in aged mice suggesting that impaired mural cell function is an important aspect of the pathology. Using a therapeutic intervention that corrects COL4A1 misfolding and secretion prevents pathology in mouse models and may represent a therapeutic avenue.

\section{Does the heart matter for your white matter? Novel cardiac markers of dementia risk}

\author{
Jodi D. Edwards
}

Atrial fibrillation (AF) is the most common cardiac arrhythmia [57] and a major cardiac cause of stroke [58]. AF is also associated with an estimated 33\% increased risk of dementia independent of stroke [59]. However, since AF is frequently intermittent or clinically silent, existing prediction tools are limited for identifying those at high risk of AF-related stroke and dementia. Left atrial cardiopathy is the process of structural and electrical remodeling preceding clinically manifest AF. Markers of left atrial cardiopathy including increased P-wave terminal in lead $\mathrm{V}_{1}$, left atrial enlargement, and excessive atrial ectopy increase the risk of $\mathrm{AF}$ and have recently been identified as a direct risk factor for stroke in the absence of AF [60]. Further, preliminary studies have shown that left atrial cardiomyopathy may also increase the risk of cognitive decline [61], suggesting that detection of atrial cardiopathy might represent an important opportunity to improve the estimation of dementia risk in aging adults. This workshop reviews evidence for atrial cardiomyopathy as a novel cardiac marker of stroke and dementia risk and highlights ongoing studies in the Brain Heart Nexus Research Program examining these and other novel markers of dementia risk.

\section{Exercise, brain health, and Alzheimer's disease}

\section{Jeff Burns}

Physical exercise may represent an important strategy for preventing, delaying the onset, or slowing the progression of AD in older adults. Our clinical and translational research suggest exercise-relevant metabolic abnormalities are present in the earliest clinical stages of $\mathrm{AD}$ [62]. We have reported that individuals in the earliest clinical stages of $\mathrm{AD}$ have alterations in muscle mass [63], bone density [64, 65], and peak oxygen consumption (VO2peak) [66-68] while these alterations in metabolic measures correlate with the degree of brain atrophy or cognitive decline. Our randomized controlled trial data suggests aerobic exercise may counteract some of these changes and influence brain structure and function in older adults both with [69] 
and without $\mathrm{AD}$ [70]. In early $\mathrm{AD}$, exercise-related increases in V02 peak are associated with improved memory performance and reduced hippocampal atrophy over 6 months [69]. In older adults without cognitive impairment, a dose-response relationship likely exists between aerobic exercise and cognition, beginning with low doses ( $75 \mathrm{~min} /$ week) and with apparent increased benefits at higher doses in those who adhered to the exercise protocol [70]. The benefits of exercise for brain health do not appear to be related to mitigation of cerebral amyloid. In a 1-year RCT of exercise vs stretching $(n=110)$, we found no exercise-related effect on cerebral amyloid levels (amyloid positron emission tomography) in healthy unimpaired adults with subclinical or elevated cerebral amyloid [71]. Our data suggests a cardiorespiratory fitness response appears to be important for brain benefits and thus maximizing an individual's cardiorespiratory fitness may be an important therapeutic target for achieving cognitive benefits.

\section{Remote ischemic conditioning for white matter health}

\section{Askiel Bruno}

Intermittent compression of the extremities with a blood pressure cuff inflated above the systolic blood pressure has been reported to exert remote vascular benefits, such as protection against subsequent heart and brain ischemia [72]. This intervention has been labeled remote ischemic conditioning (RIC) and has been usually applied daily to both arms with four cycles of 5 min alternating compression and decompression. Impressive vascular benefits from RIC have been reported in animal studies [73]. Many human RIC studies also show promising results $[74,75]$. The mechanism by which RIC leads to vascular benefits is not yet completely understood and includes neural spinal pathways and various humoral factors leading to improved blood flow to multiple tissues. The associated humoral factors include nitric oxide, various ant-inflammatory, and various neuroprotective factors. RIC is a safe and inexpensive intervention that appears promising to improve the cerebral circulation and consequently possibly protect against age-related cerebral white matter damage. RIC warrants further scientific clinical investigation.

\section{New information learned and future small Albert White Matter and Cognition workshops}

This year's Albert Research Institute workshop provided the exchange of new white matter and cognition knowledge on several fronts that include but not limited to the following:

- Changes in gut microbiota can be involved in brain tau phosphorylation and dementia.

- Female sex and adiposity can increase cognitive loss. Aging increases oxidative stress and brain microvascular endothelial dysfunction to impair NVC and contribute to cognitive decline. NVC has an essential role in maintenance of healthy cognitive function.

- Advanced age tortuosity reduces perfusion-flow velocity of cortical capillaries and white matter.

- E3 ubiquitin ligase TRIM47 is involved in agerelated PVC cerebrovascular remodeling that contributes to the pathogenesis of WMH and injury.

- OPC loss/injury is critical to white matter injury and most importantly to its repair.

- Associations between vascular risk factors across diverse populations can facilitate understanding of disease mechanisms in susceptible populations and point us to potential intervention targets.

- $\mathrm{NAD}^{+}$intermediates have potential as effective interventions in patients at risk for VCID.

- High complement factor levels of plasma EDE in aging, vascular risk, or white matter disease are associated with deficits in cognitive processing speed and executive function.

- Spatial transcriptomic work confirmed increased white matter and cortex-hippocampus senescent cells in aged mice. The BCL-2/BCL-xL inhibitor Navitoclax improves functional hyperemia and cognitive performance in aged mice.

- Damage to the blood vessels of the primitive brain can result in damage to the frontal systems resulting in executive dysfunction, gait slowing, and apathy.

- Hypertension is a highly prevalent and treatable cause of small vessel disease and VCID. LA can be an early warning system for cognitive impairment and stroke.

- Increased PIGF perivascular immunostaining occurs in the white matter CSVD. Plasma exo- 
some work indicated astrocytes and endothelial cells were the PIGF source.

- Col4al mutant mice indicate that impaired mural cell function is an important aspect of the VCID pathology. Therapeutic correction of COL4A1 misfolding and secretion prevents pathology.

- Atrial cardiomyopathy is a novel cardiac marker of stroke and dementia risk.

- Cardiorespiratory fitness can be therapeutic and provide cognitive benefits in $\mathrm{AD}$.

- RIC is a safe and inexpensive intervention to improve the cerebral circulation and protect against age-related cerebral white matter damage.

One recurrent meeting theme was the importance of age-related endothelial cell phenotypic changes that lead to dysregulation of cerebral blood flow and $\mathrm{BBB}$ disruption that promotes the pathogenesis of VCID. Vascular risk factors and systemic innate immune activation are associated with endothelial injury and inflammation that contributes to impaired $\mathrm{BBB}$ and NVC that is followed by CNS immune activation and neurodegeneration. The participants identified a need for discussions of the VCID and white matter animal models currently used and those that need to be developed. Since this first virtual annual workshop allowed the virtual attendance of participant-presenter lab members and junior scientists/fellows, although the highly effective small workshop model perpetuated in the Leo and Anne Albert Trust-sponsored scientific meetings, the pandemic has opened new virtual connections to extend communications and interactions/growth in future mixed live-virtual future workshop settings.

\section{References}

1. Roseborough A, Hachinski V, Whitehead S. White matter degeneration - a treatable target? JAMA Neurol [Internet]. 2020 Apr 27;77(7):793-4. Available from: https://jaman etwork.com/journals/jamaneurology/fullarticle/2764333

2. Barone FC, Gustafson D, Crystal HA, Moreno H, Adamski MG, Arai K, et al. First translational "Think Tank" on cerebrovascular disease, cognitive impairment and dementia. J Transl Med. 2016;14(1):1-14.

3. Sorond FA, Whitehead S, Arai K, Arnold D, Carmichael ST, De Carli C, et al. Proceedings from the Albert Charitable Trust Inaugural Workshop on white matter and cognition in aging. GeroScience. 2020;42(1):81-96.
4. Ross WC. A Public Health Policy. [Internet]. Vol. 60, The Indian medical gazette. 1925. Available from: https:// www.who.int/mental_health/publications/dementia_ report_2012/en/

5. Merkel PA, Mahr AD. Classification and epidemiology of vasculitis. Rheumatol Sixth Ed. 2014;2-2(4):1271-9.

6. Black S, Gao F, Bilbao J. Understanding white matter disease: imaging-pathological correlations in vascular cognitive impairment. In: Stroke. 2009. p. S48-52.

7. Iadecola $\mathrm{C}$. The pathobiology of vascular dementia. Neuron [Internet]. 2013;80(4):844-66. Available from: https:// doi.org/10.1016/j.neuron.2013.10.008

8. Kalaria RN. Neuropathological diagnosis of vascular cognitive impairment and vascular dementia with implications for Alzheimer's disease. Acta Neuropathol. 2016;131(5):659-85.

9. Filley CM. White matter and behavioral neurology. In: Annals of the New York Academy of Sciences. 2005. p. 162-83.

10. Filley CM. White matter dementia. White Matter Dement. 2016;5(5):1-224.

11. Mandell DM, Han JS, Poublanc J, Crawley AP, Kassner A, Fisher JA, et al. Selective reduction of blood flow to white matter during hypercapnia corresponds with leukoaraiosis. Stroke. 2008;39(7):1993-8.

12. Wang Y, Liu G, Hong D, Chen F, Ji X, Cao G. White matter injury in ischemic stroke. Prog Neurobiol [Internet]. 2016;141:45-60. Available from: https://doi.org/10. 1016/j.pneurobio.2016.04.005

13. Iadecola C, Park L, Capone C. Threats to the mind: aging, amyloid, and hypertension. Stroke [Internet]. 2009;40(3 SUPPL. 1):S40-4. Available from: http://stroke.ahajo urnals.org/content/40/3_suppl_1/S40.abstract

14. Lin J, Wang D, Lan L, Fan Y. Multiple factors involved in the pathogenesis of white matter lesions. Biomed Res Int [Internet]. 2017;2017:1-9. Available from: https://www. hindawi.com/journals/bmri/2017/9372050/

15. Markus HS, Lythgoe DJ, Ostegaard L, O'Sullivan M, Williams SCR. Reduced cerebral blood flow in white matter in ischaemic leukoaraiosis demonstrated using quantitative exogenous contrast based perfusion MRI. J Neurol Neurosurg Psychiatry. 2000;69(1):48-53.

16. Lee KM, Bang JH, Han JS, Kim BY, Lee IS, Kang HW, et al. Cardiotonic pill attenuates white matter and hippocampal damage via inhibiting microglial activation and downregulating ERK and p38 MAPK signaling in chronic cerebral hypoperfused rat. BMC Complement Altern Med. 2013;13:334.

17. Fowler JH, McQueen J, Holland PR, Manso Y, Marangoni M, Scott F, et al. Dimethyl fumarate improves white matter function following severe hypoperfusion: Involvement of microglia/macrophages and inflammatory mediators. J Cereb Blood Flow Metab [Internet]. 2018;38(8):1354-70. Available from: dimetyl fumarate; white matter

18. Qin C, Fan WH, Liu Q, Shang K, Murugan M, Wu LJ, et al. Fingolimod protects against ischemic white matter damage by modulating microglia toward M2 polarization via STAT3 pathway. Stroke. 2017;48(12):3336-46.

19. Manso Y, Holland PR, Kitamura A, Szymkowiak S, Duncombe J, Hennessy E, et al. Minocycline reduces 
microgliosis and improves subcortical white matter function in a model of cerebral vascular disease. Glia. 2018;66(1):34-46.

20. Miyanohara J, Kakae M, Nagayasu K, Nakagawa T, Mori Y, Arai K, et al. TRPM2 channel aggravates CNS inflammation and cognitive impairment via activation of microglia in chronic cerebral hypoperfusion. J Neurosci. 2018;38(14):3520-33.

21. Liu Y, Wu XM, Luo QQ, Huang S, Yang QWQ, Wang FX, et al. CX3CL1/CX3CR1-mediated microglia activation plays a detrimental role in ischemic mice brain via p38MAPK/PKC pathway. J Cereb Blood Flow Metab. 2015;35(10):1623-31.

22. Hou X, Liang X, Chen JF, Zheng J. Ecto-5'-nucleotidase (CD73) is involved in chronic cerebral hypoperfusioninduced white matter lesions and cognitive impairment by regulating glial cell activation and pro-inflammatory cytokines. Neuroscience. 2015;297:118-26.

23. Debette S, Markus HS. The clinical importance of white matter hyperintensities on brain magnetic resonance imaging: systematic review and meta-analysis. BMJ [Internet]. 2010;341(7767):288. Available from: http://www.ncbi. nlm.nih.gov/pubmed/20660506

24. Wen W, Sachdev PS, Li JJ, Chen X, Anstey KJ. White matter hyperintensities in the forties: their prevalence and topography in an epidemiological sample aged 44-48. Hum Brain Mapp [Internet]. 2009 Apr;30(4):1155-67. Available from: http://www.ncbi.nlm.nih.gov/pubmed/ 18465744

25. Roseborough AD, Langdon KD, Hammond R, Cipriano LE, Pasternak SH, Whitehead SN, et al. Post-mortem 7 Tesla MRI detection of white matter hyperintensities: a multidisciplinary voxel-wise comparison of imaging and histological correlates. NeuroImage Clin [Internet]. 2020;27(July):102340. Available from: https://doi.org/10. 1016/j.nicl.2020.102340

26. Benedetto B, Rupprecht R. Targeting glia cells: novel perspectives for the treatment of neuropsychiatric diseases. Curr Neuropharmacol. 2013;11(2):171-85.

27. Ahmed S, Gull A, Khuroo T, Aqil M, Sultana Y. Glial cell: a potential target for cellular and drug based therapy in various CNS diseases. Curr Pharm Des. 2017 Jun;23(16).

28. Prokop S, Miller KR, Heppner FL. Microglia actions in Alzheimer's disease. Acta Neuropathol. 2013;126(4):461-77.

29. Girouard H, Iadecola C. Neurovascular coupling in the normal brain and in hypertension, stroke, and Alzheimer disease. J Appl Physiol [Internet]. 2006;100(1):328-35. Available from: http://www.ncbi.nlm.nih.gov/pubmed/ 16357086

30. Korczyn AD. Mixed dementia - the most common cause of dementia. Ann N Y Acad Sci [Internet]. 2002;977(1):129-34. Available from: http://www.ncbi. nlm.nih.gov/pubmed/12480742

31. Kirova AM, Bays RB, Lagalwar S. Working memory and executive function decline across normal aging, mild cognitive impairment, and Alzheimer's disease. Biomed Res Int. 2015;2015:1-9.

32. Guarino A, Favieri F, Boncompagni I, Agostini F, Cantone M, Casagrande M. Executive functions in Alzheimer disease: a systematic review. Front Aging Neurosci. 2019;10:437.

33. Cloutier S, Chertkow H, Kergoat MJ, Gauthier S, Belleville S. Patterns of cognitive decline prior to dementia in persons with mild cognitive impairment. J Alzheimer's Dis. 2015;47(4):901-13.

34. Zlokovic B V. Neurovascular pathways to neurodegeneration in Alzheimer's disease and other disorders. Nat Rev Neurosci [Internet]. 2011;12(12):723-38. Available from: http://www.ncbi.nlm.nih.gov/pubmed/22048062

35. Faraco G, Brea D, Garcia-Bonilla L, Wang G, Racchumi G, Chang $\mathrm{H}$, et al. Dietary salt promotes neurovascular and cognitive dysfunction through a gutinitiated TH17 response. Nat Neurosci [Internet]. 2018;21(2):240-9. Available from: https://doi.org/10. 1038/s41593-017-0059-z

36. Faraco G, Hochrainer K, Segarra SG, Schaeffer S, Santisteban MM, Menon A, et al. Dietary salt promotes cognitive impairment through tau phosphorylation. Nature [Internet]. 2019;574(7780):686-90. Available from: https://doi.org/10.1038/s41586-019-1688-z

37. Salinero AE, Robison LS, Gannon OJ, Riccio D, Mansour F, Abi-Ghanem C, et al. Sex-specific effects of high-fat diet on cognitive impairment in a mouse model of VCID. FASEB J. 2020;34(11):15108-22.

38. Robison LS, Gannon OJ, Thomas MA, Salinero AE, AbiGhanem C, Poitelon Y, et al. Role of sex and high-fat diet in metabolic and hypothalamic disturbances in the 3xTgAD mouse model of Alzheimer's disease. J Neuroinflammation. 2020;17(1):285.

39. Robison LS, Albert NM, Camargo LA, Anderson BM, Salinero AE, Riccio DA, et al. High-fat diet-induced obesity causes sex-specific deficits in adult hippocampal neurogenesis in mice. eNeuro. 2020;7(1).

40. Schmidt R, Seiler S, Loitfelder M. Longitudinal change of small-vessel disease-related brain abnormalities. J Cereb Blood Flow Metab. 2016;36(1):26-39.

41. Croall ID, Lohner V, Moynihan B, Khan U, Hassan A, O'Brien JT, et al. Using DTI to assess white matter microstructure in cerebral small vessel disease (SVD) in multicentre studies. Clin Sci. 2017;131(12):1361-73.

42. Franklin RJM, Frisén J, Lyons DA. Revisiting remyelination: towards a consensus on the regeneration of CNS myelin. Semin Cell Dev Biol. 2021;116(June):3-9.

43. Sozmen EG, Rosenzweig S, Llorente IL, DiTullio DJ, Machnicki M, Vinters HV, et al. Nogo receptor blockade overcomes remyelination failure after white matter stroke and stimulates functional recovery in aged mice. Proc Natl Acad Sci U S A. 2016;113(52):E8453-62.

44. Sozmen EG, DiTullio DJ, Rosenzweig S, Hinman JD, Bridges SP, Marin MA, et al. White matter stroke induces a unique oligo-astrocyte niche that inhibits recovery. $\mathrm{J}$ Neurosci. 2019;39(47):9343-59.

45. Persyn E, Hanscombe KB, Howson JMM, Lewis CM, Traylor M, Markus HS. Genome-wide association study of MRI markers of cerebral small vessel disease in 42,310 participants. Nat Commun [Internet]. 2020;11(1):1-12. Available from: https://doi.org/10.1038/ s41467-020-15932-3

46. Fornage M, Debette S, Bis JC, Schmidt H, Ikram MA, Dufouil C, et al. Genome-wide association 
studies of cerebral white matter lesion burden. Ann Neurol. 2011;69(6):928-39.

47. Gustafson D. Epidemiology informs randomized clinical trials of cognitive impairments and late-onset, sporadic dementias. J Neurol Neuromedicine. 2018;3(5):13-8.

48. Arnoldussen IAC, Gustafson DR, Leijsen EMC, de Leeuw FE, Kiliaan AJ. Adiposity is related to cerebrovascular and brain volumetry outcomes in the RUN DMC study. Neurology [Internet]. 2019 Aug 27;93(9):e864-78. Available from: http://n.neurology.org/content/93/9/e864.abstr act

49. Bhattacharyya P, W Bynum JP, Carrillo M, Davis R, Gustafson D, Karlawish J, et al. Cost-effective early detection of cognitive decline. 2017; Available from: https://www. nia.nih.gov/sites/default/files/2018-01/final-cognitivedecline-summary.pdf

50. Tarantini S, Hertelendy P, Tucsek Z, Valcarcel-Ares MN, Smith N, Menyhart A, et al. Pharmacologically-induced neurovascular uncoupling is associated with cognitive impairment in mice. J Cereb Blood Flow Metab. 2015;35(11):1871-81.

51. Tarantini S, Tran CHT, Gordon GR, Ungvari Z, Csiszar A. Impaired neurovascular coupling in aging and Alzheimer's disease: contribution of astrocyte dysfunction and endothelial impairment to cognitive decline. Exp Gerontol [Internet]. 2017;94:52-8. Available from: https://doi.org/ 10.1016/j.exger.2016.11.004

52. Csiszar A, Tarantini S, Yabluchanskiy A, Balasubramanian P, Kiss T, Farkas E, et al. Role of endothelial NAD+ deficiency in age-related vascular dysfunction. Am J Physiol - Hear Circ Physiol. 2019;316(6):H1253-66.

53. Toth P, Tarantini S, Csiszar A, Ungvari Z. Functional vascular contributions to cognitive impairment and dementia: Mechanisms and consequences of cerebral autoregulatory dysfunction, endothelial impairment, and neurovascular uncoupling in aging. Am J Physiol - Hear Circ Physiol. 2017;312(1):H1-20.

54. Ungvari Z, Tarantini S, Sorond F, Merkely B, Csiszar A. Mechanisms of vascular aging, a geroscience perspective: JACC Focus Seminar. J Am Coll Cardiol. 2020;75(8):931-41.

55. Ungvari Z, Tarantini S, Kiss T, Wren JD, Giles CB, Griffin CT, et al. Endothelial dysfunction and angiogenesis impairment in the ageing vasculature. Nat Rev Cardiol [Internet]. 2018;15(9):555-65. Available from: https://doi. org/10.1038/s41569-018-0030-Z

56. Yabluchanskiy A, Tarantini S, Balasubramanian P, Kiss T, Csipo T, Fülöp GA, et al. Pharmacological or genetic depletion of senescent astrocytes prevents whole brain irradiation-induced impairment of neurovascular coupling responses protecting cognitive function in mice. GeroScience. 2020;42(2):409-28.

57. Lippi G, Sanchis-Gomar F, Cervellin G. Global epidemiology of atrial fibrillation: An increasing epidemic and public health challenge. Int J Stroke. 2021;16(2):217-21.

58. Wolf PA, Abbott RD, Kannel WB. Atrial fibrillation as an independent risk factor for stroke: The framingham study. Stroke. 1991;22(8):983-8.

59. De Bruijn RFAG, Heeringa J, Wolters FJ, Franco $\mathrm{OH}$, Stricker BHC, Hofman A, et al. Association between atrial fibrillation and dementia in the general population. JAMA Neurol. 2015;72(11):1288-94.

60. Edwards JD, Healey JS, Fang J, Yip K, Gladstone DJ. Atrial cardiopathy in the absence of atrial fibrillation increases risk of ischemic stroke, incident atrial fibrillation, and mortality and improves stroke risk prediction. J Am Heart Assoc. 2020;9(11).

61. Alosco ML, Gunstad J, Jerskey BA, Clark US, Hassenstab JJ, Xu X, et al. Left atrial size is independently associated with cognitive function. Int $\mathrm{J}$ Neurosci. 2013;123(8):544-52.

62. Morris JK, Honea RA, Vidoni ED, Swerdlow RH, Burns JM. Is Alzheimer's disease a systemic disease? Biochim Biophys Acta - Mol Basis Dis [Internet]. 2014;1842(9):1340-9. Available from: https://doi.org/10. 1016/j.bbadis.2014.04.012

63. Burns JM, Johnson DK, Watts A, Swerdlow RH, Brooks WM. Reduced lean mass in early Alzheimer disease and its association with brain atrophy. Arch Neurol. 2010;67(4):428-33.

64. Loskutova N, Honea RA, Brooks WM, Burns JM. Reduced limbic and hypothalamic volumes correlate with bone density in early Alzheimer's disease. J Alzheimer's Dis. 2010;20(1):313-22.

65. Loskutova N, Honea RA, Vidoni ED, Brooks WM, Burns JM. Bone density and brain atrophy in early Alzheimer's disease. J Alzheimer's Dis. 2009;18(4):777-85.

66. Burns JM, Cronk BB, Anderson HS, Donnelly JE, Thomas GP, Harsha A, et al. Cardiorespiratory fitness and brain atrophy in early Alzheimer disease. Neurology [Internet]. 2008 Jul 15;71(3):210-6. Available from: http://n.neurology.org/content/71/3/210.abstract

67. Honea RA, Thomas GP, Harsha A, Anderson HS, Donnelly JE, Brooks WM, et al. Cardiorespiratory fitness and preserved medial temporal lobe volume in alzheimer disease. Alzheimer Dis Assoc Disord. 2009;23(3):188-97.

68. Vidoni ED, Honea RA, Billinger SA, Swerdlow RH, Burns JM. Cardiorespiratory fitness is associated with atrophy in Alzheimer's and aging over 2 years. Neurobiol Aging [Internet]. 2012;33(8):1624-32. Available from: https://doi.org/10.1016/j.neurobiolaging.2011.03.016

69. Morris JK, Vidoni ED, Johnson DK, Van Sciver A, Mahnken JD, Honea RA, et al. Aerobic exercise for Alzheimer's disease: a randomized controlled pilot trial. PLoS ONE. 2017;12(2):1-14.

70. Vidoni ED, Johnson DK, Morris JK, Van Sciver A, Greer CS, Billinger SA, et al. Dose-response of aerobic exercise on cognition: a community-based, pilot randomized controlled trial. PLoS ONE. 2015;10(7):1-13.

71. Vidoni ED, Morris JK, Watts A, Perry M, Clutton J, van Sciver A, et al. Effect of aerobic exercise on amyloid accumulation in preclinical Alzheimer's: a 1-year randomized controlled trial. PLoS One [Internet]. 2021;16(1 January):1-18. Available from: https://doi.org/10.1371/ journal.pone.0244893

72. Zhou D, Ding J, Ya J, Pan L, Wang Y, Ji X, et al. Remote ischemic conditioning: a promising therapeutic intervention for multi-organ protection. Aging (Albany NY). 2018;10(8):1825-55.

73. Khan MB, Hoda MN, Vaibhav K, Giri S, Wang P, Waller $\mathrm{JL}$, et al. Remote ischemic postconditioning: harnessing 
endogenous protection in a murine model of vascular cognitive impairment. Transl Stroke Res. 2015;6(1):69-77.

74. Billah M, Ridiandries A, Allahwala U, Mudaliar H, Dona A, Hunyor S, et al. Circulating mediators of remote ischemic preconditioning: search for the missing link between non-lethal ischemia and cardioprotection. Oncotarget. 2019;10(2):216-44.

75. Zhou D, Ding J, Ya J, Pan L, Bai C, Guan J, et al. Efficacy of remote ischemic conditioning on improving WMHs and cognition in very elderly patients with intracranial atherosclerotic stenosis. Aging (Albany NY). 2019;11(2):634-48.

Publisher's note Springer Nature remains neutral with regard to jurisdictional claims in published maps and institutional affiliations. 